\title{
Kinetic Monte Carlo Simulations of dislocations in heteroepitaxial growth
}

\author{
F. Much*, M. Ahr, M. Biehl and W. Kinzel \\ Sonderforschungsbereich 410 \\ Institut für Theoretische Physik \\ Julius-Maximilians-Universität Würzburg \\ Am Hubland, 97074 Würzburg, Germany
}

October 30, 2018

\begin{abstract}
We determine the critical layer thickness for the appearance of misfit dislocations as a function of the misfit $\varepsilon$ between the lattice constants of the substrate and the adsorbate from Kinetic Monte Carlo (KMC) simulations of heteroepitaxial growth.

To this end, an algorithm is introduced which allows the off-lattice simulation of various phenomena observed in heteroepitaxial growth (see e.g. [1, 2]) including critical layer thickness for the appearance of misfit dislocations, or self-assembled island formation. The only parameters of the model are deposition flux, temperature and a pairwise interaction potential between the particles of the system.

Our results are compared with a theoretical treatment of the problem and show good agreement with a simple power law.
\end{abstract}

\section{Introduction}

Heteroepitaxial growth has been a field of intense study in recent years. This is mainly due to the improved performance of semiconductor and opto-electronic devices which can be achieved using strained layer epitaxy. Here the heteroepitaxial growth by depositing material onto a substrate with the same crystal structure as the adsorbate but a slightly different lattice constant is of special interest.

In the early stages of this kind of heteroepitaxial growth the adsorbate is coherent with the substrate. In this state the crystal topology is that of a perfect crystal, i.e. each particle has the same coordination number and its nearest and next-nearest neighbors form the same geometrical figure with only slightly modified distances [1, 2].

As the thickness of the adsorbate film increases the elastic energy of the film rises until it is energetically favorable to form dislocations in order to relieve the strain. In this new incoherent state the crystal topology is perturbed near the substrate/adsorbate interface. The thickness of the adsorbate film at which this occurs is called the critical layer thickness $h_{c}$. Theoretical models were proposed in order to determine $h_{c}$ as a function of material parameters [1, 23, 4, 5]. In this letter we determine the critical layer thickness as a function of

*e-mail: much@physik.uni-wuerzburg.de 
the misfit between the lattice constants of the substrate and the adsorbate using computer simulations.

In order to simulate heteroepitaxial growth one has to overcome the limitations of a fixed lattice. One obvious way to perform off-lattice simulations are molecular dynamics (MD), see for instance [四] in the context of misfit dislocations. MD methods have the advantage of conceptual simplicity but can only be applied to rather small system sizes at very high temperature and deposition rates. Because of the high computational efforts the critical layer thickness has been determined for only a few values of the misfit.

Here, we propose a KMC algorithm for the simulation of the early stages of heteroepitaxial growth. In contrast to similar off-lattice algorithms suggested before - for example by Faux et al. [6, 7], Plotz et al. [8] or Schindler [9] - we are able to simulate heteroepitaxial growth for rather thick adsorbate layers and over a wide range of the misfit between the lattice constants of the substrate and the adsorbate.

In the following we consider only growth in $1+1$ dimensions. However, the method can be extended to $2+1$ dimensional growth. To our knowledge this is the first time that the critical layer thickness for the appearance of dislocations is observed in Monte Carlo simulations. We

find that our results fit well to a power law, as it has been observed for several semiconductor compounds [5].

\section{Method}

The aim here is to gain general insight into relevant mechanisms of heteroepitaxial growth. We therefore choose a simple Lennard-Jones potential

$$
U_{i j}(\sigma)=4 U_{0}\left[\left(\frac{\sigma}{r_{i j}}\right)^{12}-\left(\frac{\sigma}{r_{i j}}\right)^{6}\right] .
$$

as particle interaction, which is numerically easy to handle and saves computer time compared to more realistic empirical potentials like the EAM approximation (see e.g. [10]). However, we focus on the observation of effects which should not depend on the particular choice of the potential.

The equilibrium distance $r_{0}$ between two isolated particles interacting via $U_{i j}$ becomes $r_{0}=\sqrt[6]{2} \sigma$ and is slightly smaller in the bulk material. Because of the isotropy of the LennardJones potential the particles arrange in a triangular lattice. In order to save computer time the interaction potential $U_{i j}$ is cut off at a distance $r_{i j}>3 r_{0}$. The interaction strength at this distance is less than $1 \%$ of the value at the equilibrium distance and can therefore be neglected. The interaction of two substrate particles is given by $U_{i j}\left(\sigma_{S}\right)$. Two adsorbate particles interact via $U_{i j}\left(\sigma_{A}\right)$ whereas we assume that a substrate and an adsorbate particle interact via $\frac{1}{2}\left(U_{i j}\left(\sigma_{S}\right)+U_{i j}\left(\sigma_{A}\right)\right)$.

We would like to stress that growth is not simulated on a fixed lattice but rather two particles $i$ and $j$ are separated by a continuous distance $r_{i j}$. There are two possible events in our simulations: deposition and diffusion of adsorbate particles. The two-dimensional simulation cell is open in vertical and has periodic boundary conditions in lateral direction. Adsorbate particles are randomly deposited on the crystal surface with a rate $R_{d}=L F$, where $L$ is the system size and $F=1 s^{-1}$ is the deposition flux. The rate $R_{i}$ for a diffusion event $i$ is given by an Arrhenius law

$$
R_{i}=\nu_{0} e^{-\frac{E_{a, i}}{k_{B} T}}
$$


where $\nu_{0}=10^{12} s^{-1}, E_{a, i}, T$ are the attempt frequency, the activation barrier for the diffusion step $i$ and the simulation temperature, respectively.

$E_{a, i}$ is given by $E_{a, i}=E_{t, i}-E_{b, i}$, where $E_{t, i}$ denotes the energy of the particle at the transition state and $E_{b, i}$ the energy at the binding state. Both are calculated for a frozen crystal using Brent's method [11]. Here the saddle point search for the calculation of $E_{t, i}$ can be replaced by a simpler maximum search in $1+1$ dimensions.

To consider the elastic deformation of the crystal after each microscopic event (diffusion or deposition) the total potential energy of the $n$ particles - system

$$
E_{t o t}=\sum_{i=1}^{n} \sum_{j=i+1}^{n} U_{i j}
$$

is minimized using a conjugate gradient method [11] under variation of the coordinates of all particles (substrate and adsorbate) within a circle of radius $3 r_{0}$ around the particle where the event took place. In order to avoid strain caused by this local relaxation of the crystal, after a distinct number of microscopic events - depending on the misfit $\varepsilon$ - a minimization of $E_{t o t}$ under variation of all particle coordinates is performed.

The obtained rates for deposition and diffusion of adsorbate particles are used in a rejection free KMC simulation. Using a binary tree structure [12] an event $i$ is chosen with the correct probability $R_{i} / R$, where

$$
R=R_{d}+\sum_{i} R_{i}
$$

is the total rate of all microscopic processes. Then this event is performed and the rates of all affected events are updated. Unlike in standard Monte Carlo simulations time does not advance linearly in discrete time steps $\Delta t$. Instead the time interval $\tau$ between two microscopic processes is randomly drawn from a Poisson distribution $P(\tau)=R e^{-R \tau}$ by $\tau=-\frac{\ln \rho}{R}$, where $\rho$ is a uniformly distributed random number between 0 and 1 .

Each simulation run starts with six atomic layers of substrate with a fixed bottom layer. The system size $L$ (number of particles in the substrate's upper layer) is between $L=100$ and $L=200$. Within this range we found no significant dependence of the results on $L$. Measuring lengths in units of $\sigma_{S}, \sigma_{A}$ is chosen between 0.85 and 1.11 , so we can simulate heteroepitaxial growth for misfits

$$
\varepsilon=\frac{\sigma_{A}-\sigma_{S}}{\sigma_{S}}
$$

between $-15 \%$ and $+11 \%$.

\section{Results and Discussion}

In the following the temperature is set to $T=0.03 \frac{U_{0}}{k_{B}}$, which is sufficiently low compared to the melting temperature $T_{m}=0.415 \frac{U_{0}}{k_{B}}$ of a pure two-dimensional Lennard-Jones solid [13].

Heteroepitaxial growth is now simulated in order to determine the critical layer thickness $h_{c}$ for the appearance of dislocations as a function of the misfit $\varepsilon$.

To this end between 5 and 10 independent simulation runs are carried out for each value of $\varepsilon$. We find that in each simulation run several dislocations appear simultaneously. Then, after the deposition of a few monolayers of adsorbate after the first appearance of a dislocations in the crystal the number of dislocations remains constant. The thickness of the adsorbate layer at which dislocations first appear is registered as $h_{c}$. 

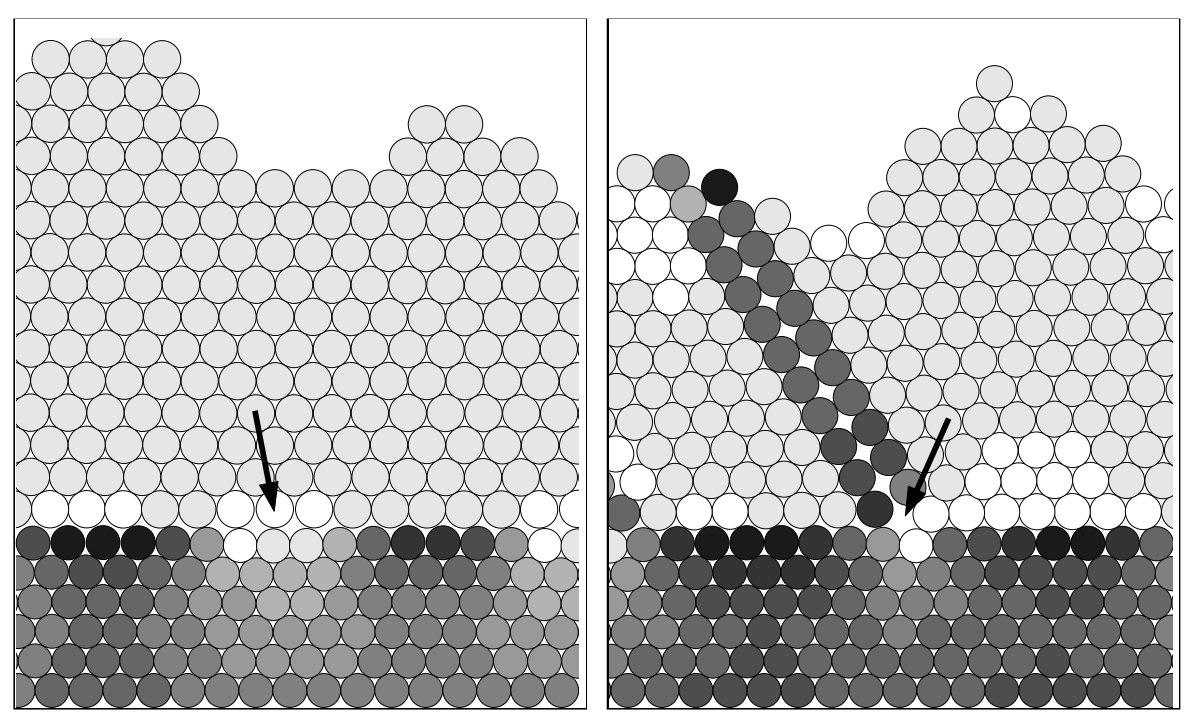

Figure 1: Typical sections of crystals obtained in our simulations. The six bottom layers are the given substrate. The dislocations are marked with arrows. Left panel: perfect dislocation for $\varepsilon=+10 \%$. Right panel: partial dislocation for $\varepsilon=+6 \%$. The grey level for a particle indicates the particle's average distance to its nearest neighbors of the same kind: the lighter its grey level the more is this particle under compression.

To prove the existence of a dislocation we determine the coordination number $n_{c}$ of each particle by calculating the Voronoy polyhedra 14, 15]. Voronoy polyhedra are a generalization of the Wigner-Seitz cell to a system without a fixed lattice. The number of sides of a Voronoy polyhedron gives the coordination number $n_{c}\left(n_{c}=6\right.$ for a particle in a perfect triangular lattice). A Burgers circuit [16] is drawn around regions of the crystal with $n_{c} \neq 6$. A nonvanishing Burgers vector then indicates the appearance of a dislocation.

Figure 1 shows sections of two crystals obtained in our simulations for left panel $\varepsilon=+10 \%$ and right panel $\varepsilon=+6 \%$. The grey level for a particle in these pictures is obtained from the particle's average distance to its nearest neighbors of the same kind. The lighter the grey level the more is this particle under compression.

\subsection{Number of Dislocations}

Figure 2 shows the number of dislocations per unit length $n_{D} / L$ counted for each value of $\varepsilon$ about 6 monolayers deposited adsorbate after the first appearance of a dislocation in the crystal (the maximum number of dislocations should be reached for this thickness of the adsorbate layer). The dashed line gives the theoretical number of dislocations in a system of size $L$ under the assumption that $n_{D}=L|\varepsilon|$ perfect dislocations can appear. Perfect dislocations (fig. 1 left panel) are those for which the crystal topology far from the substrate/adsorbate interface is the same as in the coherent state and the Burgers vector is therefore an integer multiple of the lattice vector. The formation of partial dislocations (fig. 11 right panel) characterized by a Burgers vector which is a rational fraction of a lattice vector - causes the deviations from the theoretical results for $-0.07 \leq \varepsilon \leq-0.03$ and $0.04 \leq \varepsilon \leq 0.08$. This is due to the fact, that partial dislocations are spatially more extended than perfect dislocations. For this reason in the case $\varepsilon>0$ more and for $\varepsilon<0$ less dislocations than $n_{D}=L|\varepsilon|$ have to 


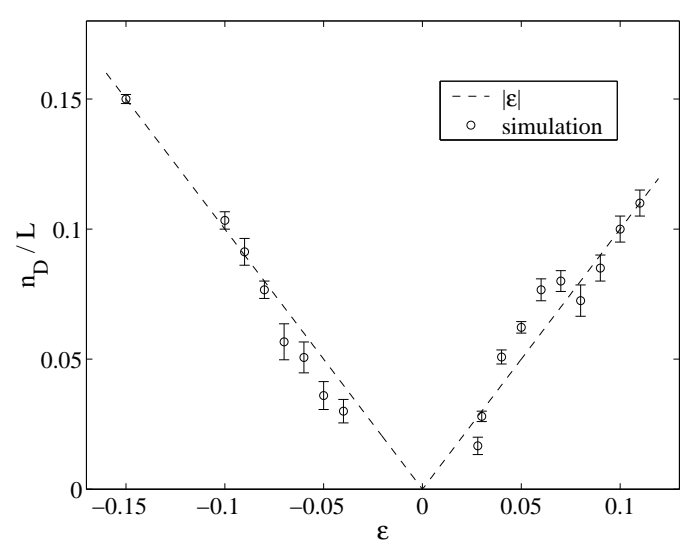

Figure 2: Number of dislocations per system size $n_{D} / L$ as a function of the misfit $\varepsilon$. The error bars represent as the standard error of the simulation results. The dashed line gives the theoretical number of perfect dislocations in a system of size $L$.

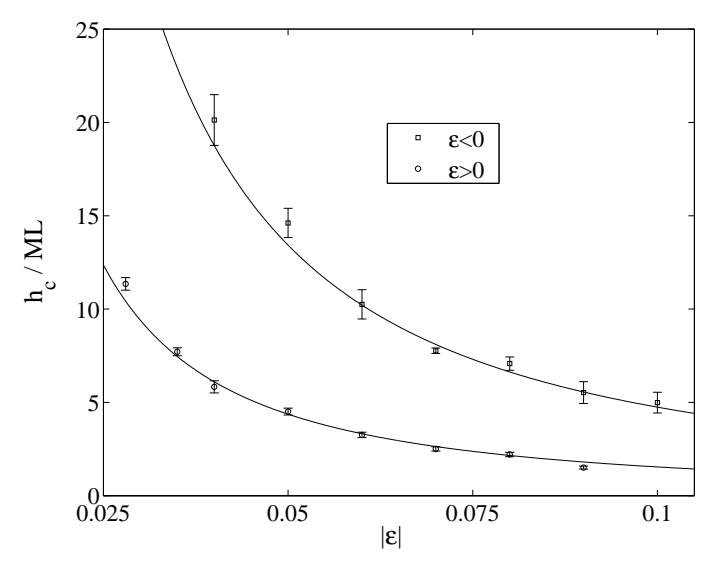

Figure 3: Critical thickness $h_{c}$ versus misfit $|\varepsilon|$ for $\varepsilon<0$ (upper curve) and $\varepsilon>0$ (lower curve). The error bars are obtained as the standard error of the simulation results. The solid lines are calculated using eq. (6) where $a^{*}=0.15$ for $\varepsilon<0$ and $a^{*}=0.05$ for $\varepsilon>0$.

be built when partial dislocations appear. Why partial dislocations only appear for distinct values of $\varepsilon$ is still unknown.

\subsection{Critical layer thickness}

Figure 3 shows the critical layer thickness $h_{c}$ plotted versus the absolute value of the misfit $\varepsilon$. For $-0.03<\varepsilon<0.02$ the critical thickness is too large to be observed in our simulations. The simulation results show a dependence of $h_{c}$ on the sign of the misfit. This was found before by L. Dong et al. [4]. We believe this dependence is due to the fact, that the LennardJones potential is not harmonic. The potential is steeper in compression $(\varepsilon>0)$ than in tension $(\varepsilon<0)$, so that for $\varepsilon>0$ it becomes favorable to form a dislocation for smaller values of $h_{c}$.

Our simulation results agree well with a power law (solid lines in fig. 3)

$$
h_{c}=a^{*} \varepsilon^{-3 / 2}
$$

which was proposed by Cohen-Solal et al. [5].

There, an energy balance model is proposed for calculating the critical layer thickness in heteroepitaxial growth of semiconductor compounds. To this end the classical strain energy, without any change of the substrate or dislocation formation, and the deformation energy due to a full system of interfacial misfit dislocations were compared. The method yields the $\varepsilon^{-3 / 2}$ power law, where $h_{c}$ depends mainly on the misfit $\varepsilon$. Their model was compared with experimental data revealing an excellent agreement for IV-IV, III-V and II-VI semiconductor compounds with values for $a^{*}$ between $a^{*}=0.15$ and $a^{*}=0.50$. A nonlinear fit of our results yields $a^{*}=0.15$ for $\varepsilon<0$ and $a^{*}=0.05$ for $\varepsilon>0$ 


\section{Conclusion}

The goal of this examination was to determine the critical layer thickness for heteroepitaxial growth of Lennard-Jones particles. For this purpose we propose a novel KMC algorithm. We demonstrate, that with this algorithm it is possible to simulate the appearance of dislocations for a wide range of misfits and rather large system sizes compared to commonly used methods like molecular dynamics simulations.

We find our simulation data in good agreement with a very simple $\varepsilon^{-3 / 2}$ power law, where the critical thickness depends mainly on the lattice mismatch. This law has been identified in various systems before [5] and may thus be considered as quite general.

The developed algorithm is applicable in the simulation of various other phenomena observed in heteroepitaxial growth. In future work we will examine the 2D-3D transition in island growth and step-bunching on vicinal surfaces, for instance.

We would like to thank A. Schindler for fruitful discussions about problems concerning

the simulation of heteroepitaxial growth. FM and MA were supported by the Deutsche Forschungsgemeinschaft.

\section{References}

[1] Politi P., Grenet G., Marty A., Ponchet A. and Villain J., Phys. Rep. 324 271, 2000

[2] Pimpinelli A. and Villain J., Physics of Crystal Growth, Cambridge University Press, 1998

[3] Matthews J. W. and Blakeslee A. E., J. Crystal Growth 27 118, 1974

[4] Dong L., Schnitker J., Smith R. W. and Sroloviz D. J., J. Appl. Phys. 83 217, 1997

[5] Cohen-Solal G., Bailly F. and Barbé M., J. Crystal Growth 138 68, 1994

[6] Faux D. A., Gaynor G., Carson C. L., Hall C. K. and Bernholc J., Phys. Rev. B 422914 , 1990

[7] Spjut H. and Faux D. A., Surf. Sc. 306 233, 1994

[8] Plotz W. M., Hingerl K. and Sitter H., Phys. Rev. B 45 12122, 1992

[9] Schindler A., Theoretical aspects of growth on one and two dimensional strained crystal surfaces, Dissertation, Duisburg 1999

[10] Somfai E. and Sander L. M., Strain in heteroepitaxial growth, cond-mat/9909328, 1999

[11] Press W.H., Teukolsky S. A., Vetterling W. T. and Flannery B. P., Numerical Recipes in $C$, Cambridge University Press, 1992

[12] Newman M. E. J. and Barkema G. T., Monte Carlo Methods in Statistical Physics, Oxford University Press, 1999

[13] F.F. Abraham, in Melting, Localization, and Chaos, edited by R.K. Kalia and P. Vashishta. Elsevier, New York, 1982. 
[14] N.W. Ashcroft, N.D. Mermin. Solid State Physics. Saunders College Publishing, 1976.

[15] K. J. Strandburg. Rev. Modern Physics 60(1) 1988

[16] J. P. Hirth, J. Lothe, Theory of Dislocations. McGraw-Hill Book Company, 1968. 